\title{
Cyclotron-synchrotron: Harmonic fitting functions in the non-relativistic and trans-relativistic regimes
}

\author{
A. Marcowith ${ }^{1}$ and J. Malzac ${ }^{2,3}$ \\ 1 C.E.S.R., 9 Av. du Colonnel Roche, 31028 Toulouse Cedex 4, France \\ 2 Institute of Astronomy, Madingley Road, Cambridge, CB3 0HA, UK \\ 3 Osservatorio di Brera, via Brera, 28, 20121 Milano, Italy \\ e-mail:malzac@ast.cam.ac.uk
}

Received 14 April 2003 / Accepted 30 June 2003

\begin{abstract}
The present work investigates the calculation of absorption and emission cyclotron line profiles in the non-relativistic and trans-relativistic regimes. We provide fits for the ten first harmonics with synthetic functions down to $10^{-4}$ of the maximum flux with an accuracy of $20 \%$ at worst. The lines at a given particle energy are calculated from the integration of the Schott formula over the photon and the particle solid angles relative to the magnetic field direction. The method can easily be extended to a larger number of harmonics. We also derive spectral fits of thermal emission line plasmas at non-relativistic and transrelativistic temperatures extending previous parameterisations.
\end{abstract}

Key words. line: profiles - magnetic fields - radiation mechanisms: non-thermal, thermal

\section{Introduction}

The cyclotron-synchrotron radiation is produced by charged particles spiraling in a magnetic field. The non-relativistic limit (for particle speed $\beta=v / c \ll 1$ ) is the cyclotron radiation. The relativistic limit (for particle speed $\beta=v / c \sim 1$ ) is the synchrotron radiation. At intermediate speeds, the process is sometimes called gyro-magnetic (for $\beta=v / c \sim 0.5$ ).

The cyclotron-synchrotron effect is one of the most important processes in astrophysics and has been invoked in solar, neutron star physics and in galactic or extra-galactic jets. At non relativistic and ultra relativistic limits, the particle emissivity is provided by well known analytical formulae.

The main contribution to the cyclotron radiation comes from low harmonics. In most astrophysical objects, this radiation is either self-absorbed or absorbed by the environment. It can even be suppressed by plasma effects such as in the Razin-Tsytovich effect. However, as stressed by different authors (Ghisellini \& Svensson 1991, hereafter GS91; Mason 1992; Gliozzi et al. 1996) the cyclotron cross-section in the non- and mildly relativistic regimes is several orders of magnitude above the Thompson cross section. The cyclotron mechanism appears then as a very efficient thermalisation process in hot plasmas surrounding compact objects. At higher energies, the synchrotron photons are a supplementary source for the Comptonisation process in accretion discs coronæ, see e.g. Di Matteo et al. (1997), Ghisellini et al. (1998), hereafter

Send offprint requests to: A. Marcowith, e-mail: Alexandre. Marcowith@cesr.fr
GHS98, Wardziński \& Zdziarski (2000), hereafter WZ00 and Wardziński \& Zdziarski (2001).

A detailed numerical investigation of the thermalisation process in the presence of cyclo-synchrotron photons has scarcely been discussed (see however GHS98) but is of great importance for the spectral and temporal modeling of the complex spectral energy distribution produced in compact objects. Low temperature plasmas that may be found in gamma-ray bursts (in the comoving frame) and in accretion discs may produce cyclotron lines combined with synchrotron signatures. Such line features can be produced in neutron star magnetospheres probing the magnetic field.

The present work aims to propose useful fitting formulae for the first ten harmonics dominating the emissivity for particle velocities (or normalised momentum) between $\beta=$ 0.01 and $\mathrm{p}=\beta \gamma=2$ (see Fig. 2 in Mahadevan et al. 1996 hereafter MNY96). We consider the case of an isotropic electron distribution in a tangled magnetic field. The method can be extended to an arbitrary number of harmonics. At higher energies, however, the synchrotron formulae are worth being used. The derived functions can then be easily inserted into radiative transfer codes.

The article is organised as follows: Sect. 2 recalls the derivation of cyclo-synchrotron emissivity. Section 3 deals with the procedures used to compute the emission and the absorption coefficient: an integration over a small frequency interval around the cyclotron resonant frequency and a direct integration over the angle between the photon and the magnetic field. These procedures are tested in Sect. 4. Section 5 provides the 
fitting functions in the non-thermal case, at a given particle energy. Section 6 provides the fitting functions for the emission coefficients produced in a thermal plasma.

\section{Cyclo-synchrotron emissivity}

The cyclo-synchrotron spectrum produced by one particle of mass $m$, charge $e$ and velocity $v=\beta \cdot c$ embedded in a uniform magnetic field $B$ can be derived from classical electrodynamics (see for example Bekefi 1966, chapter 6). The emitted power spectrum, the so-called Schott formula is expressed as a sum of harmonics peaking at a frequency $v=n v_{\mathrm{b}} / \gamma(n \geq 1)$, where $\gamma=\left(1-\beta^{2}\right)^{-1 / 2}$ is the Lorentz factor of the particle. The cyclotron frequency is $v_{\mathrm{b}}=e B /(2 \pi m c)$, where the magnetic field $B$ is expressed in Gauss units.

$\frac{\mathrm{d} P}{\mathrm{~d} v \mathrm{~d} \Omega}=2 \pi \frac{e^{2}}{c} v^{2} \sum_{n=1}^{\infty}\left[\frac{\left(\cos \theta-\beta_{\|}\right)^{2}}{\sin ^{2} \theta} J_{n}^{2}(x)+\beta_{\perp}^{2} J_{n}^{\prime 2}(x)\right] \delta(y)$,

with $x=v \gamma \beta_{\perp} \sin \theta / \nu_{\mathrm{b}}, \beta_{\|}=\beta \cos \theta_{\mathrm{p}}, \beta_{\perp}=\beta \sin \theta_{\mathrm{p}}$ and $J_{n}, J_{n}^{\prime}$ being the Bessel functions of (integer) order $n$ and its first derivatives.

The cyclotron resonance is defined by

$y=n \frac{\nu_{\mathrm{b}}}{\gamma}-v\left(1-\beta_{\|} \cos \theta\right)=0$.

Equation (1) is expressed in CGS units (e.g. $\operatorname{erg~s}^{-1} \mathrm{~Hz}^{-1} \mathrm{st}^{-1}$ ) as well as all equations in the text.

The angles are defined relative to the dominant magnetic field direction: $\theta_{\mathrm{p}}$ is the particle pitch-angle and $\theta$ is the photon angle.

Integrating Eq. (1) over the photon angle and the frequency, averaging the pitch-angle leads to the total power emitted by one particle

$P_{\mathrm{tot}}=\frac{4}{3} \sigma_{\mathrm{T}} c(\gamma \beta)^{2} \frac{B^{2}}{8 \pi}$,

where the Thomson cross section is $\sigma_{\mathrm{T}} \simeq 6.65 \times 10^{-25} \mathrm{~cm}^{2}$.

At the non-relativistic limit, only the first harmonics contribute significantly to the emissivity. For the particular pitchangle $\theta_{\mathrm{p}}=\pi / 2$, and after integration over all observer angles, the power emitted in a given harmonic $n$ is (Bekefi 1966)

$\eta_{n}=\frac{8 \pi^{2} e^{2} v_{\mathrm{b}}^{2}}{c} \frac{(n+1)\left(n^{2 n+1}\right)}{(2 n+1) !} \beta^{2 n}$.

The ratio of two successive harmonics scales roughly as $\beta^{2}$.

In the relativistic limit $(\beta \rightarrow 1)$ the emissivity is dominated by the harmonics of order $n \sim \gamma^{2}$. The spectrum tends towards a continuum as the frequency interval between two harmonics is $v_{\mathrm{b}} / \gamma$. The synchrotron power radiated by one particle per unit frequency interval is given by (we still assume $\theta_{\mathrm{p}}=\pi / 2$ )

$$
\begin{aligned}
\frac{\mathrm{d} P}{\mathrm{~d} v} & =\frac{\sqrt{3} e^{2} v_{\mathrm{b}}}{c} \frac{v}{v_{\mathrm{c}}} \int_{\frac{v}{v_{\mathrm{c}}}}^{\infty} K_{5 / 3}(t) \mathrm{d} t \\
& \sim \frac{\sqrt{3} e^{2} v_{\mathrm{b}}}{c} 1.8 x^{0.3} \exp (-x),
\end{aligned}
$$

where the critical frequency is $v_{\mathrm{c}}=3 / 2 v_{\mathrm{b}} \gamma^{2}, x=v / v_{\mathrm{c}}$ and $K_{5 / 3}(t)$ is the modified Bessel function of order $5 / 3$. The particle emissivity scales as $v^{1 / 3}$ for $x \ll 0.29$ ( 0.29 is the energy of maximum emissivity) and is exponentially cut-off at high energies (Ginzburg \& Syrovatskii 1969).

\section{Calculation procedures}

We developed two methods to numerically estimate the cyclotron emissivity and absorption cross-section of one particle at a given energy and frequency. Each method aims to treat the resonant term inside the Dirac function in a correct way, see Eqs. (1) and (2).

\subsection{Frequency integration}

The first procedure consists in integrating the expression (1) over $v$ in a narrow frequency range $[v-\Delta v / 2, v+\Delta v / 2]$, with $\Delta v=\alpha v \ll v, \alpha$ being a positive real variable.

Equation (1) becomes

$$
\begin{aligned}
\frac{\mathrm{d} P}{\mathrm{~d} v \mathrm{~d} \Omega}\left(\theta_{\mathrm{p}}\right) \sim & \frac{2 \pi e^{2} v_{\mathrm{b}}^{2}}{\alpha v c} \sum_{n=n_{1}}^{n_{2}} \frac{n^{2}}{\gamma^{2}\left(1-\beta_{\|} \cos \theta\right)^{3}} \\
& \times\left[\left(\frac{\cos \theta-\beta_{\|}}{\sin \theta}\right)^{2} J_{n}^{2}\left(x_{\mathrm{r}}\right)+\beta_{\perp}^{2} J_{n}^{\prime 2}\left(x_{\mathrm{r}}\right)\right] .
\end{aligned}
$$

The resonant term is $x_{\mathrm{r}}=n \beta_{\perp} \sin \theta /\left(1-\beta_{\|} \cos \theta\right)$. The limits $n_{1}$ and $n_{2}$ are fixed by the resonant condition (2) taken at $v-\Delta v / 2$ and $v+\Delta v / 2$.

\subsection{Direct integration}

The second procedure eliminates the resonance through a direct integration over $\theta$, the angle between the photon and the magnetic field. The resonance condition selects an angle for a given harmonic $n$, a given pitch-angle and $\beta$

$\cos \theta_{\mathrm{r}}=\frac{1-\frac{n v_{\mathrm{b}}}{\gamma \nu}}{\beta_{\|}}$

Integrated over the solid angle, Eq. (1) leads to

$\frac{\mathrm{d} P}{\mathrm{~d} v}\left(\theta_{\mathrm{p}}\right)=\frac{2 \pi e^{2} v}{\beta_{\|} c} \sum_{n=n_{1}}^{n_{2}}\left(\frac{\cos \theta_{\mathrm{r}}-\beta_{\|}}{\sin \theta_{\mathrm{r}}}\right)^{2} J_{n}^{2}\left(x_{\mathrm{r}}\right)+\beta_{\perp}^{2} J_{n}^{\prime 2}\left(x_{\mathrm{r}}\right)$.

The resonant term is now $x_{\mathrm{r}}=v \gamma \beta_{\perp} \sin \theta_{\mathrm{r}} / v_{\mathrm{b}}$. The sum boundaries $n_{1}$ and $n_{2}$ define the range of $n$ for which $\left|\cos \theta_{\mathrm{r}}\right| \leq 1$ in Eq. (7).

We note that for $\cos \theta_{\mathrm{p}}=0, \cos \theta_{\mathrm{r}}$ becomes undefinite and Eq. (8) breaks down. In this case, all the power is radiated at the resonant frequencies, i.e. $\mathrm{d} P / \mathrm{d} v\left(\theta_{\mathrm{p}}=\pi / 2\right)=0$ except at frequencies $v=m v_{\mathrm{b}} / \gamma$ and at those frequencies the power per unit frequency is actually infinite. The total power emitted in harmonic $n$ at pitch angle $\theta_{\mathrm{p}}=\pi / 2$ may be estimated by integrating Eq. (1) over frequencies around the resonance (leading to a result similar to Eq. (6)) and then numerically over the observer angles $\theta$. 


\subsection{Discussion: Total line emission}

The power spectrum $\mathrm{d} P /\left.\mathrm{d} v\right|_{n}$ at a given harmonic is obtained by integrating numerically Eq. (6) over the photon solid angle $\Omega$ and over the particle pitch-angle $\Omega_{\mathrm{p}}$ using an extended Simpson formula. For the direct integration in Eq. (8) only one integral over the particle pitch-angle is necessary.

Both methods fail to account for the unavoidable resonance at frequencies $v=n v_{\mathrm{b}} / \gamma$ that is due to the contribution from pitch angle $\theta_{\mathrm{p}}=\pi / 2$. In the frequency integration method, the Dirac function is artificially broadened over the integration bin of width $\alpha v$. This leads to an overestimate the line emissivity around the resonances, but the resulting frequency integrated power is conserved. In the direct integration scheme we neglected the resonant contribution at frequencies $v=n \nu_{\mathrm{b}} / \gamma$. Although this procedure gives more accurate line profiles, the frequency integrated power is slightly underestimated. The two independent methods are compared for different particle energy regimes in Fig. 1. They appear to be in agreement within $\sim 10 \%$ at low energies and $\sim 20 \%$ at high particle energies. The errors (the relative difference between the two emissivities) have been calculated down to $10^{-2}$ the line maximum. The calculated flux obtained from the second method appears to be lower around the peak of emission at least for the first few harmonics and at low particle energies. The discrepancy is always of the order of $10 \%$, less than the accuracy we aim to reach using the fitting functions. For this reason, we will only consider the first (frequency integration) method for the tests detailed in Sect. 3. For both methods, the numerical integration of the power spectrum over frequencies gives the total power (Eq. (3)) within a few percent.

The best agreements (between the two methods) were obtained using an $\alpha$ coefficient (associated with the frequency integration method) in the range $10^{-3}$ to a few times $10^{-2}$. These values ensure a stable numerical integration. MNY96 have developed a similar but different method, approximating the Dirac peak using a smooth function over a frequency interval $\alpha v$. They reach a good numerical stability with $\alpha=5 \times 10^{-2}$. In the following, we use the first method to find the fitting functions at a given particle energy (see Sect. 5), the second method has the advantage of avoiding one integration and will be used in the thermal plasma case (see Sect. 6).

\subsection{The cyclo-synchrotron absorption cross section}

The cyclo-synchrotron absorption cross section has been derived by GS91 from the Einstein coefficients in a three level system. The differential cross section is the difference between true absorption and stimulated emission cross sections. In the relativistic limit, the stimulated effect takes over for large photon angles with the magnetic field, but the total cross section is always positive.

If the photon energy is much smaller than the electron kinetic energy $\left(v \ll \gamma m_{\mathrm{e}} c^{2} / h\right)$, the angle integrated cross section can be expressed by the angle averaged emissivity $j(v, \beta)=$ $(\mathrm{d} P / \mathrm{d} v) / 4 \pi$ in a differential form as

$\sigma(\nu, \beta)=\frac{1}{2 m_{\mathrm{e}} v^{2}} \frac{1}{\gamma p} \frac{\partial[\gamma p j(\nu, \beta)]}{\partial \gamma}$.
The cyclo-synchrotron cross section can reach values $\sim 10^{16} \sigma_{\mathrm{T}} / B_{\mathrm{G}}$ for $v=v_{\mathrm{b}}$, and $\beta \rightarrow 0$.

The absorption cross section at a given frequency for $a$ given harmonic ( $n_{0} \leq 10, \beta$ and $v$ are fixed) is calculated using Eq. (6). While integrating over the solid angles, we first test if the harmonic $n_{0}$ is in the range of the permitted harmonics evaluated at a given pair of angles $\theta, \theta_{\mathrm{p}}$. The harmonic number interval is defined from Eq. (2) by

$n_{1}=E\left[(1-\alpha) v \gamma\left(1-\beta_{\|} \cos \theta\right)\right]+1$,

$n_{2}=E\left[(1+\alpha) v \gamma\left(1-\beta_{\|} \cos \theta\right)\right]$.

Once the test is successfully passed, we calculate the analytical derivative over $\gamma$ of the emissivity at the harmonic number $n_{0}$, e.g. the derivative of

$$
\begin{aligned}
\left.\frac{\mathrm{d} P}{\mathrm{~d} v \mathrm{~d} \Omega}\right|_{n_{0}} \sim & \frac{2 \pi e^{2} v_{\mathrm{b}}^{2}}{\alpha v c} \frac{n_{0}^{2}}{\gamma^{2}\left(1-\beta_{\|} \cos \theta\right)^{3}} \\
& \times\left[\left(\frac{\cos \theta-\beta_{\|}}{\sin \theta}\right)^{2} J_{n_{0}}^{2}\left(x_{\mathrm{r}}\right)+\beta_{\perp}^{2} J_{n_{0}}^{\prime 2}\left(x_{\mathrm{r}}\right)\right],
\end{aligned}
$$

with $x_{\mathrm{r}}=n_{0} \beta_{\perp} \sin \theta /\left(1-\beta_{\|} \cos \theta\right)$. We finally sum at each frequency bin the contribution from the ten first harmonics to get $\sigma(\nu, \beta)$.

\section{Tests}

We performed a series of tests for our numerical schemes in both mono-energetic and thermal cases. The methods developed are not well adapted to the relativistic regime as the number of harmonics to be summed increases radically when $\beta \rightarrow 1$. They failed to converge to the correct analytical expression (5) at high frequencies.

We first tried to recover the known cyclotron limit, the total power radiated by one particle, at a given harmonic and compared it with expression (4). The second test compares the numerical cyclotron absorption coefficient at a given harmonic with the non-relativistic coefficient provided by GS91. For the thermal plasma case, we recover the cyclotron absorption coefficients given by Chanmugam et al. (1989) (hereafter C89) and the Kirchoff law.

\subsection{Tests for mono-energetic distribution}

\subsubsection{Cyclotron limit}

In the cyclotron limit $(\beta \rightarrow 0)$, we integrate the first harmonics over the resonant frequency range with a particle pitch-angle $\theta_{\mathrm{p}}=\pi / 2$ and compare our results with Eq. (4). We plot the relative differences for different $\beta$ for the first ten harmonics in Fig. 2. As can be seen, the agreement is quite good, even if the errors increase with the harmonic number, however they are limited to few percent.

Concerning the absorption cross section we compared our results with the analytical expressions given by GS91. We point out that their formulae have to be corrected by a factor $1 / 4 \pi$ in order to verify Eq. (9). Then, at low energy and at frequencies $\sim \nu_{\mathrm{b}}$, we did obtained a good agreement and a cross section of the order of $\sigma(v, \beta \rightarrow 0) \sim 10^{16} B_{\mathrm{G}}^{-1} \sigma_{\mathrm{T}}$. In the trans-relativistic 

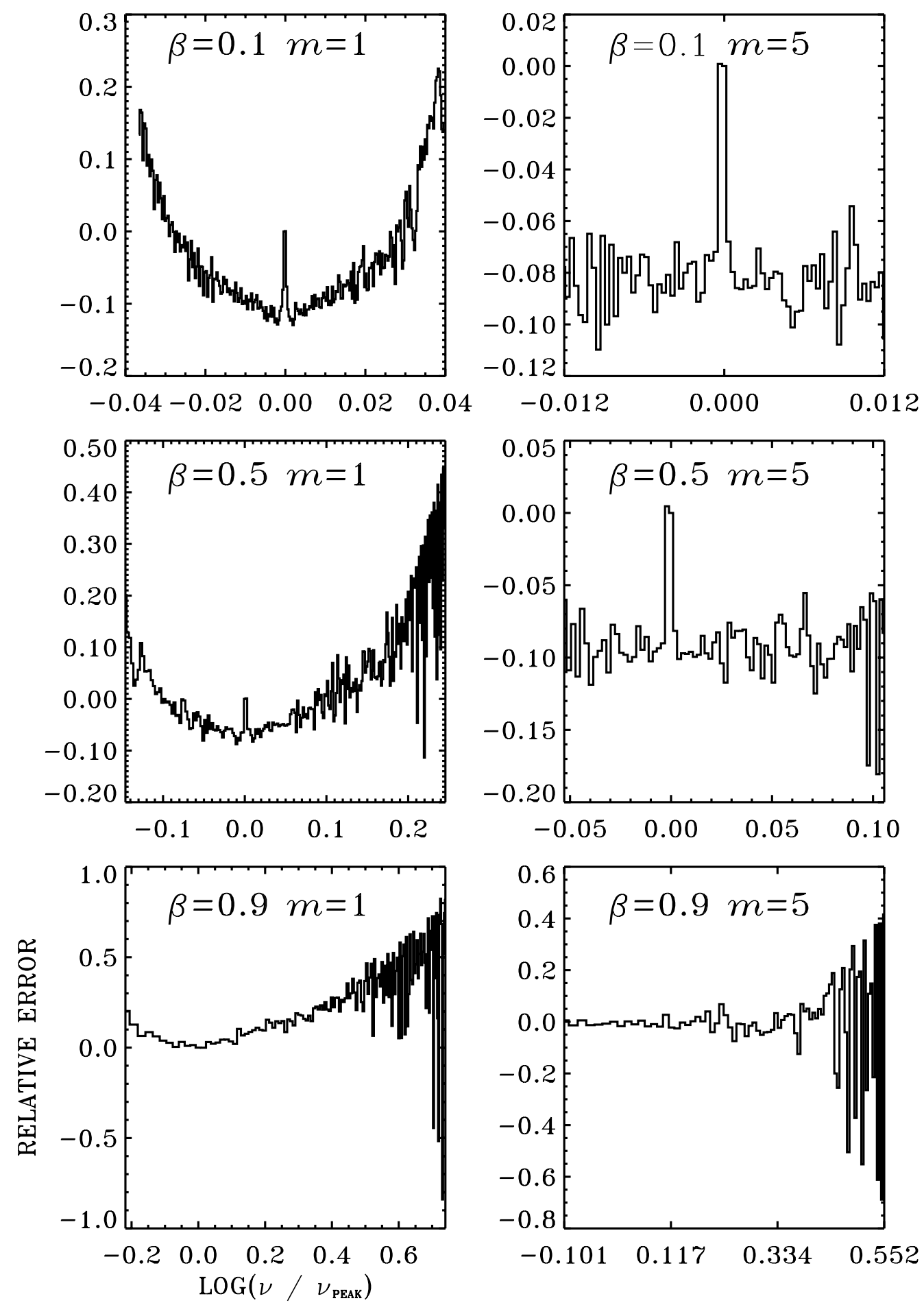

Fig. 1. Relative differences between the two formulae (6) and (8) deriving the power emitted by one particle. The energy is scaled to the line peak energy. The harmonics 1 and 5 are shown at three particle energies $\beta=0.1,0.5,0.9$ corresponding to the energy ranges explored in this work.

regime, we also found a good agreement at frequencies $v \geq v_{\mathrm{b}}$ (see Fig. 8, lower panel).

\subsection{Thermal absorption coefficients}

C89 (and references therein) derived the thermal absorption coefficients for non relativistic thermal plasmas $(T \leq 50 \mathrm{keV})$ for the two polarisation modes. The ordinary mode has an electric vector field oriented along the magnetic field given by the first term in Eq. (1), while the extraordinary mode has an electric vector field perpendicular to the magnetic field given by the second term in Eq. (1). The extraordinary mode usually dominates. At different harmonics, we calculate the absorption coefficients from the emissivity at a given angle $\theta$ integrated over 


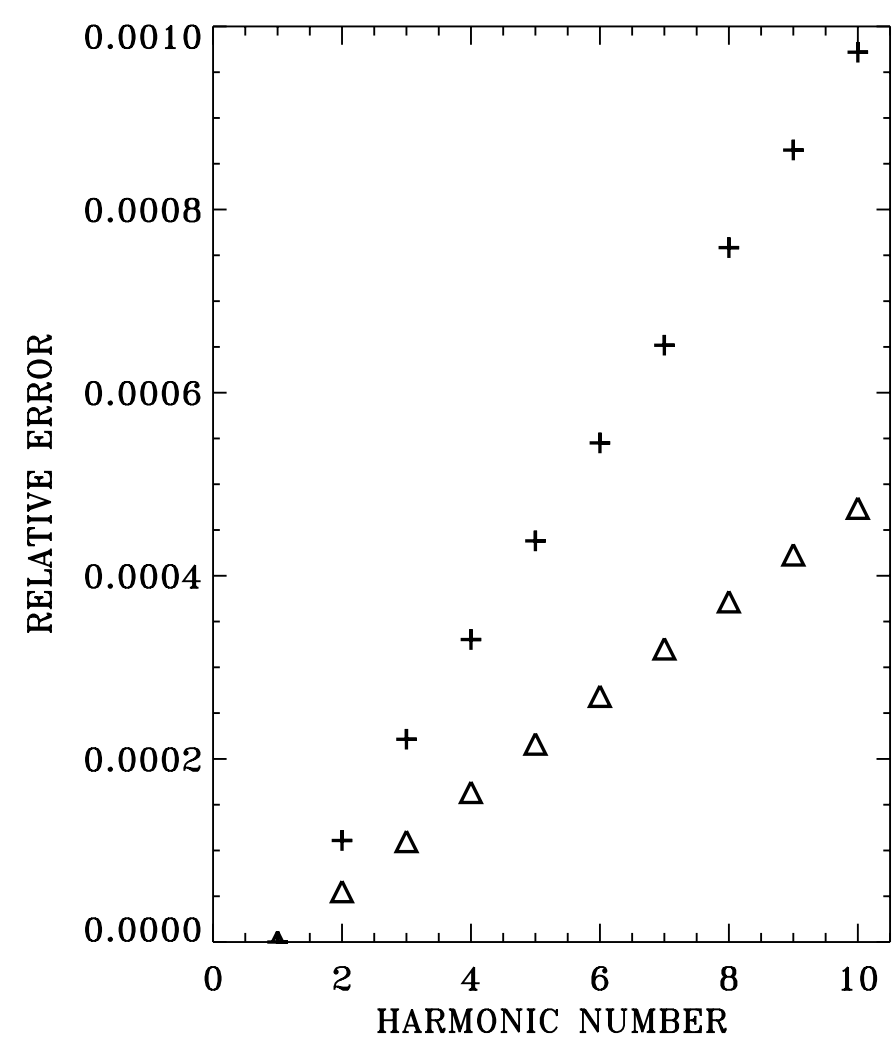

Fig. 2. Relative differences between Eq. (6), the power emitted by one particle integrated over the solid angle and Eq. (4), the power radiated by one particle in the non-relativistic regime. Both emissivities have been calculated at a pitch-angle $\theta_{\mathrm{p}}=\pi / 2$. The plot is provided for the ten first harmonics and for two different particle energies $\beta=10^{-2}$ (triangles), and 0.1 (crosses). The emissivities have been normalised to the harmonic one. For scaling reasons, the errors have been divided by 50 for the $\beta=0.1$ case.

a Maxwellian distribution $N_{\mathrm{e}} /\left(\Theta K_{2}(1 / \Theta)\right) \gamma^{2} \beta \exp (-\gamma / \Theta)$. We have defined the dimensionless temperature as $\Theta=k_{B} T / m c^{2}$ and $N_{\mathrm{e}}$ the electron density.

We plot our results in Fig. 3 for three temperatures at three different $\theta$ values and at different frequencies covering the first 20 harmonics. The agreement with $\mathrm{C} 89$ is good (see their Tables $6 \mathrm{~A}, 6 \mathrm{C}$ and $6 \mathrm{E}$ ), the numerical thermal coefficient falls close to the extraordinary mode values.

Using the same calculation procedure, we check that both thermal emission and absorption coefficients fulfill the Kirchoff law, $j_{v}=B_{v}(T) \alpha_{v}$. We plot, in Fig. 4, the relative differences between $j_{v} / \alpha_{v}$ obtained from numerical integration with $B_{v}(T)$ versus the frequency for non-relativistic temperatures. The agreement is again good except for $T \geq 10 \mathrm{keV}$ at $v \sim v_{\mathrm{b}}$ where the flux is weak and the uncertainties obtained with the numerical integration method increase.

\section{Non-thermal lines}

We now describe the fitting function for the mono-energetic case in the non- and trans-relativistic regimes. Whenever it was possible we used simple (essentially polynomial) functions defined by the smallest number of parameters. Accounting for the evolution of the line profile with the particle energy,
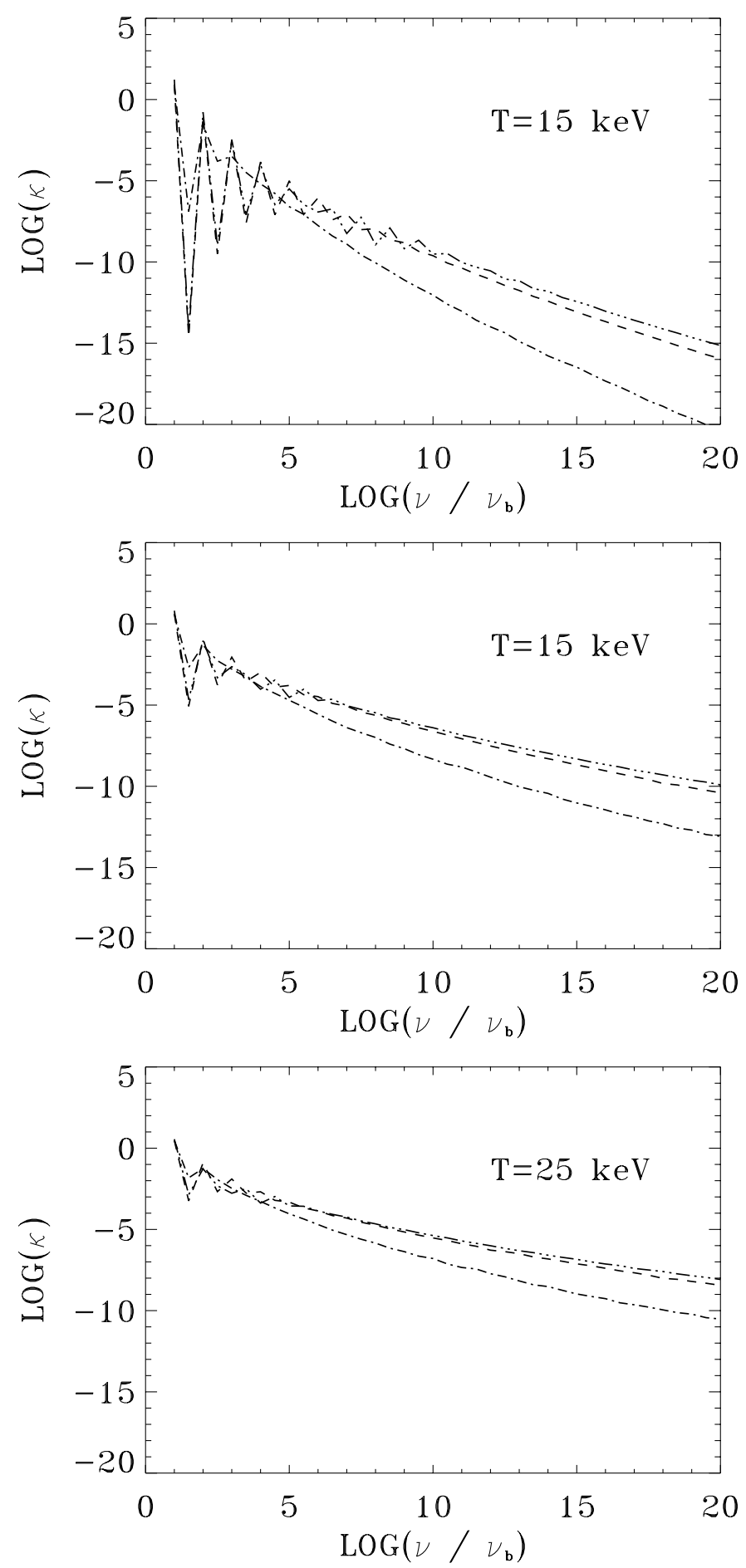

Fig. 3. Thermal absorption coefficients (in $\mathrm{cm}^{-1}$ ) at $T=5,15,25 \mathrm{keV}$. The photon angles are $\theta=80^{\circ}$ three dotted-dashed line, $60^{\circ}$ longdashed line, $30^{\circ}$ dot-dashed line. The results can be compared with C89. The calculations have been performed for a magnetic field $B=$ 10 MG.

we split our parameters into two classes: the parameters below the energy of the line peak, the parameters above the energy of the line peak. The line normalisation with $\beta$ and $n$ is also parametrised. 


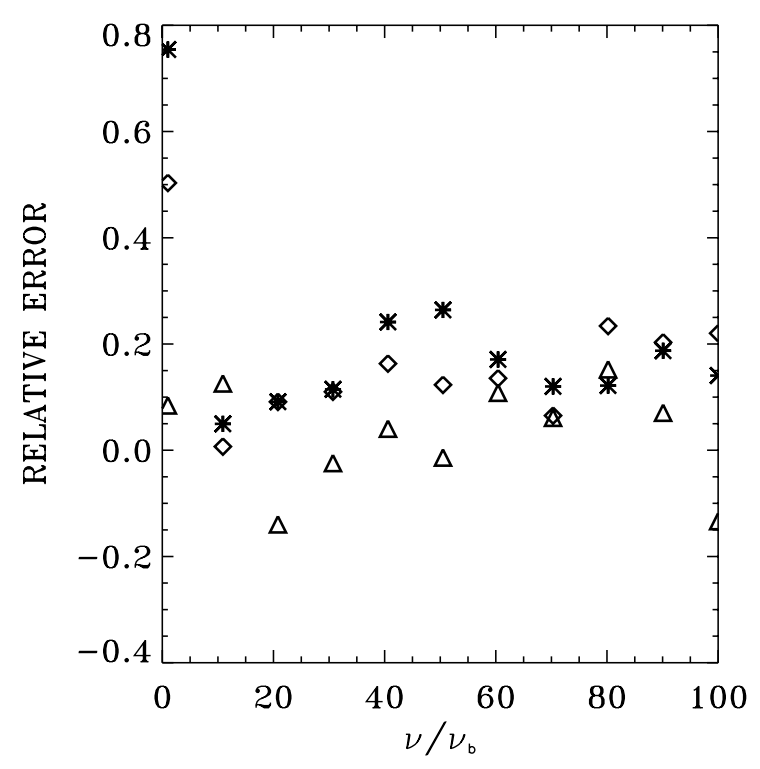

Fig. 4. Relative differences between the ratio of the calculated emission and absorption coefficients $j_{v} / \alpha_{v}$ and $B_{v}(T)$ at non-relativistic temperatures $T=1$ (triangles), 10 (squares), 50 (stars) $\mathrm{keV}$, in the frequency range $1-100 v / v_{\mathrm{b}}$.

The generic form for the fitting functions is

$F(n, \beta, v)=N_{1}\left(\frac{K_{1}}{x^{I_{1}}+K_{1}-1}\right)^{I_{2}}$,

where

$x=\frac{v-v_{\text {inf }}}{v_{\max }-v_{\text {inf }}}$, for $v \leq v_{\max }$,

$x=\frac{v_{\text {sup }}-v}{v_{\text {sup }}-v_{\max }}$, for $v \geq v_{\max }$.

The different frequencies are $v_{\text {inf }}=v_{\mathrm{b}} n /(\gamma[1+\beta]), v_{\text {sup }}=$ $v_{\mathrm{b}} n /(\gamma[1-\beta])$ given by the resonant condition Eq. (2). The maximum frequency is $v_{\max }=v_{\mathrm{b}} n / \gamma$. This choice respects the normalisation $F\left(n, \beta, v_{\max }\right)=N_{1}$. The parameters are chosen in order for the functions (12) to fit the line (where the worst error was $20 \%$ ) in a frequency range $v_{\max }$ up (down) to a frequency $v_{\text {end }>}\left(v_{\text {end }<}\right)$ where the line flux is $10^{-4}$ the peak flux, we have the ordering $v_{\text {inf }} \leq\left(v_{\text {end }<}\right) \leq v_{\text {max }} \leq\left(v_{\text {end }>}\right) \leq v_{\text {sup }}$.

This value was chosen to ensure good flux estimates at frequencies between two lines maxima where in the transrelativistic regime, two subsequent harmonics may both contribute significantly to the emissivity.

The parameters are $N_{1}(n, \beta) \geq 0$ for the line normalisation, $K_{1}(n, \beta) \geq 0, I_{1}(n, \beta) \geq 0$ and $I_{2}(n, \beta) \geq 0$ for the line profile.

Once a line is fitted, the $n$ and $\beta$ dependencies of the four primary parameters $N_{1}, K_{1}, I_{1}, I_{2}$ have to be determined using other fitting functions. The whole fitting procedure is twofold, e.g. we first derive the previous coefficients in a grid $(\beta, n)$ using Eq. (12). We called this the primary fitting procedure. We then provide new fitting functions for both $\beta$ and $n$ dependencies of the four previous parameters, this is the secondary fitting procedure.
Obviously, the general fitting function (12) is not unique, other forms may be much more accurate for the primary fitting procedure but they usually require more parameters and lead to a complex second step. The solutions obtained are polynomials of degrees lower than 4, they are accurate enough to be used in a wide range of problems dealing with cyclotron radiation.

We consider two energy regimes: the cyclotron-gyromagnetic (non-relativistic) regime defined by $\beta \leq 0.5$ and in the trans-relativistic regime defined by $p \leq 2$ and $\beta \geq 0.5$. Beyond this, the usual relativistic formulae apply. This choice was motivated by the different behavior of the primary parameters in the two regimes (see next).

For both emission and absorption lines, the second exponent, at a given harmonic, $I_{2}(n)$ has been fixed at $\beta=0.01$ (the lowest energy considered here) and has an imposed evolution in the non relativistic regime (up to $\beta=0.5$ ). Above, in the trans relativistic regime, we re-initialise $I_{2}(n)$ to its value at $\beta=0.5$ and impose an other evolution with $\beta$. This procedure limits the fluctuations in the other two primary parameters $I_{1}$ and $K_{1}$. We also had to consider the evolution of the first harmonic individually for both emission and absorption coefficients, and the evolution of the second harmonic also individually for the absorption coefficient.

The number of parameters being high (6 parameters for the shape and one for the normalisation), the results are unfortunately too complex to be presented in tabular forms. We have therefore decided to provide fortran routines calculating the power spectrum for frequencies $v \leq 10 v_{\mathrm{b}}$. This procedure has been adopted for the thermal case too (see next section). All the programs are accessible via ftp anonymous at $\mathrm{ftp}$. cesr. $\mathrm{fr} / \mathrm{pub} /$ synchrotron (in cyclosynchro.tar.gz). We simply present here results in a graphical form.

\subsection{Results}

For each figure, the first plot presents the numerical calculation and their fits superimposed and the second plot presents the relative errors. Figures 5 and 6 display the results for both emission and absorption lines in the non-relativistic limits at particle energies corresponding to $\beta=0.1$ and $\beta=0.3$. Figures 7 and 8 display the results from the trans-relativistic regime at particle energies corresponding to $\beta=0.7$ and $\beta=0.9$.

The units are $\mathrm{cm}^{-1}$ and $\mathrm{erg} \mathrm{s}^{-1} \mathrm{~Hz}^{-1} \mathrm{~cm}^{-3} \mathrm{st}^{-1}$ for the absorption and emission coefficients respectively.

In most of the cases, the harmonics are fitted down to $10^{-4}$ the peak line flux with errors never exceeding $20 \%$ (ratio larger than 1.25 or lower than 0.8). Exceptions may be found in Figs. 5 and 6 for frequencies $v \geq v_{\max }$ at harmonic number $n \geq 4$ where the profile is very sharp. The flux is overestimated here but for a restrained frequency range. However, even in these cases errors are around $20 \%$ and the higher harmonics do not strongly contribute to the overall flux. The errors increase at the edges of a given harmonic in the non-relativistic regime and at frequencies $v$ of the order $v_{\text {inf }}$ or $v_{\text {sup }}$ in the transrelativistic regime due to the low accuracy. The noise appearing at the edge of the frequency domain $\left(1-10 v_{\mathrm{b}}\right)$ is due to the numerical integration method. As the flux decreases, the method 
would have required a frequency dependent (increasing) $\alpha$ in Eq. (6) to keep a smooth line profile. As the flux at these energies are far beyond $10^{-4}$ the peak line flux, we disregarded the problem. However the emission (or absorption) beyond $10 v_{\mathrm{b}}$ is not well reproduced since at these energies harmonics of higher order contribute and the synchrotron formula is worth to be used. Finally note that the flux in the absorption of the first harmonic in the trans-relativistic case is under-estimated by a factor two under $v_{\max }$ for fluxes lower than $10^{-2}$ the peak flux.

Also presented are the non-relativistic and the relativistic limits derived by GHS98 and GS91 respectively. In Fig. 8 one can see a good agreement at frequencies $v>v_{\mathrm{b}}$ between the numerical and the analytical calculations, for both emission and absorption coefficients.

\section{Thermal emission lines}

The previous emission coefficients are now integrated over a relativistic Maxwellian distribution at a given temperature $\Theta=k_{B} T / m_{\mathrm{e}} c^{2}$. As stated above (Sect. 3.2), for computational facilities, we used the second method (direct integration).

We computed the line spectra for the ten first harmonics at 200 different temperatures forming a logarithmic grid in the range of $\Theta=10^{-4}-1$. We make available electronically a table of emissivities in the 10 first harmonics as a function of photon frequency and a fortran routine reading this table and interpolating between the precomputed emissivities. The absorption coefficients can then be derived from the Kirchoff law.

We found that the emission due to an individual harmonic can be conveniently approximated as follows:

Let $v_{\max }$ be the photon frequency of the peak of harmonic $n$ (see previous paragraph). We define the frequencies $v_{1}$ and $v_{2}$ so that:

$$
\begin{aligned}
v_{1} & =\frac{0.999 v_{\text {max }}^{2}}{(n+\Theta) v_{\mathrm{b}}} \\
v_{2} & =(n+0.001) v_{\mathrm{b}},
\end{aligned}
$$

we have $v_{1} \leq v_{\max } \leq v_{2}$.

We introduce the following notations:

$$
\begin{aligned}
& x=v /\left(n v_{\mathrm{b}}\right), \\
& x_{1}=v_{1} /\left(n v_{\mathrm{b}}\right), \\
& x_{2}=v_{2} /\left(n v_{\mathrm{b}}\right) .
\end{aligned}
$$

Let $N_{1}, N_{2}$ and $N_{\max }$ be the line emissivity at $v_{1}, v_{2}$ and $v_{\max }$. We further note $p_{1}, p_{2}, p_{3} \ldots$ etc., the parameters depending on $n$ and $\Theta$ that provide the best fit to our numerical results. Then, for $v<v_{1}$, the thermal emission coefficient can be conveniently approximated by the function:

$$
\begin{aligned}
F(n, \Theta, v)= & N_{1}\left(\frac{v}{v_{1}}\right)^{p_{1}} \frac{1-p_{2} x_{1}}{1-p_{2} x} \\
& \times \exp \left[-\frac{p_{3}}{2 \Theta}\left(\frac{1}{x}-\frac{1}{x_{1}}+x-x 1\right)\right] .
\end{aligned}
$$

For $v_{1}<v<v_{\max }$ :

$F(n, \Theta, v)=N_{\max } \exp \left[a \ln \left(v / v_{\max }\right)^{2}+b \ln \left(v / v_{\max }\right)^{3}\right]$
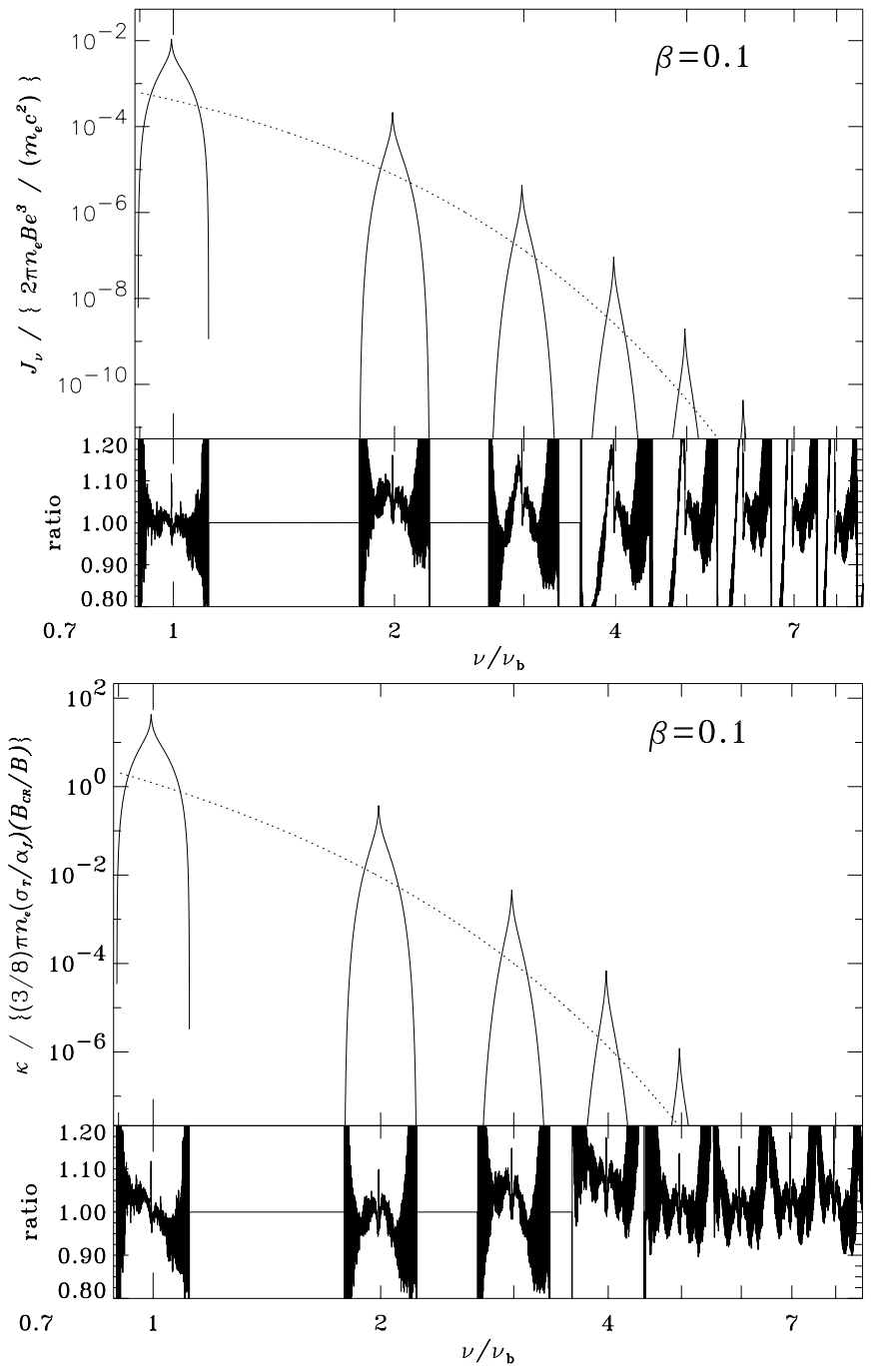

Fig. 5. Results for non-thermal lines at $\beta=0.1$. The first upper panel shows the spectra produced using the fitting functions (solid line). The flux obtained from numerical integration, indistinguishable at this scale, has been skipped for clarity. The ratio of the fitting function to the numerical results is plotted in the lower panel. The second panel shows the fits and the ratio for the absorption line. The emission and the absorption coefficients are normalised to $\left(2 \pi n_{\mathrm{e}} e B^{3}\right) /\left(m_{\mathrm{e}} c^{2}\right)$ and $3 / 8 \pi N_{\mathrm{e}}\left(\sigma_{\mathrm{T}} / \alpha_{\mathrm{f}}\right)\left(B_{\mathrm{cr}} / B\right)$ respectively $\left(\alpha_{\mathrm{f}}=1 / 137\right.$ is the fine structure constant and $B_{\text {cr }} \sim 4.4 \times 10^{13}$ Gauss is the critical magnetic field). The same normalisation is adopted for all figures. The formula (8) of GHS98 and (2.23) of GS91 in the non-relativistic limit has been used to plot the emission and absorption coefficients with a dotted line.

where:

$a=\left(3-p_{4}\right) \ln \left(N_{1} / N_{\max }\right) / \ln \left(v_{1} / v_{\max }\right)^{2}$

$b=\left(p_{4}-2\right) \ln \left(N_{1} / N_{\max }\right) / \ln \left(v_{1} / v_{\max }\right)^{3}$.

For $v_{\max }<v<v_{2}$ :

$F(n, \Theta, v)=N_{\max } \exp \left[c \ln \left(v / v_{\max }\right)^{2}+d \ln \left(v / v_{\max }\right)^{3}\right]$

where:

$c=\left(3-p_{5}\right) \ln \left(N_{2} / N_{\max }\right) / \ln \left(v_{2} / \nu_{\max }\right)^{2}$

$d=\left(p_{5}-2\right) \ln \left(N_{2} / N_{\max }\right) / \ln \left(v_{2} / v_{\max }\right)^{3}$. 

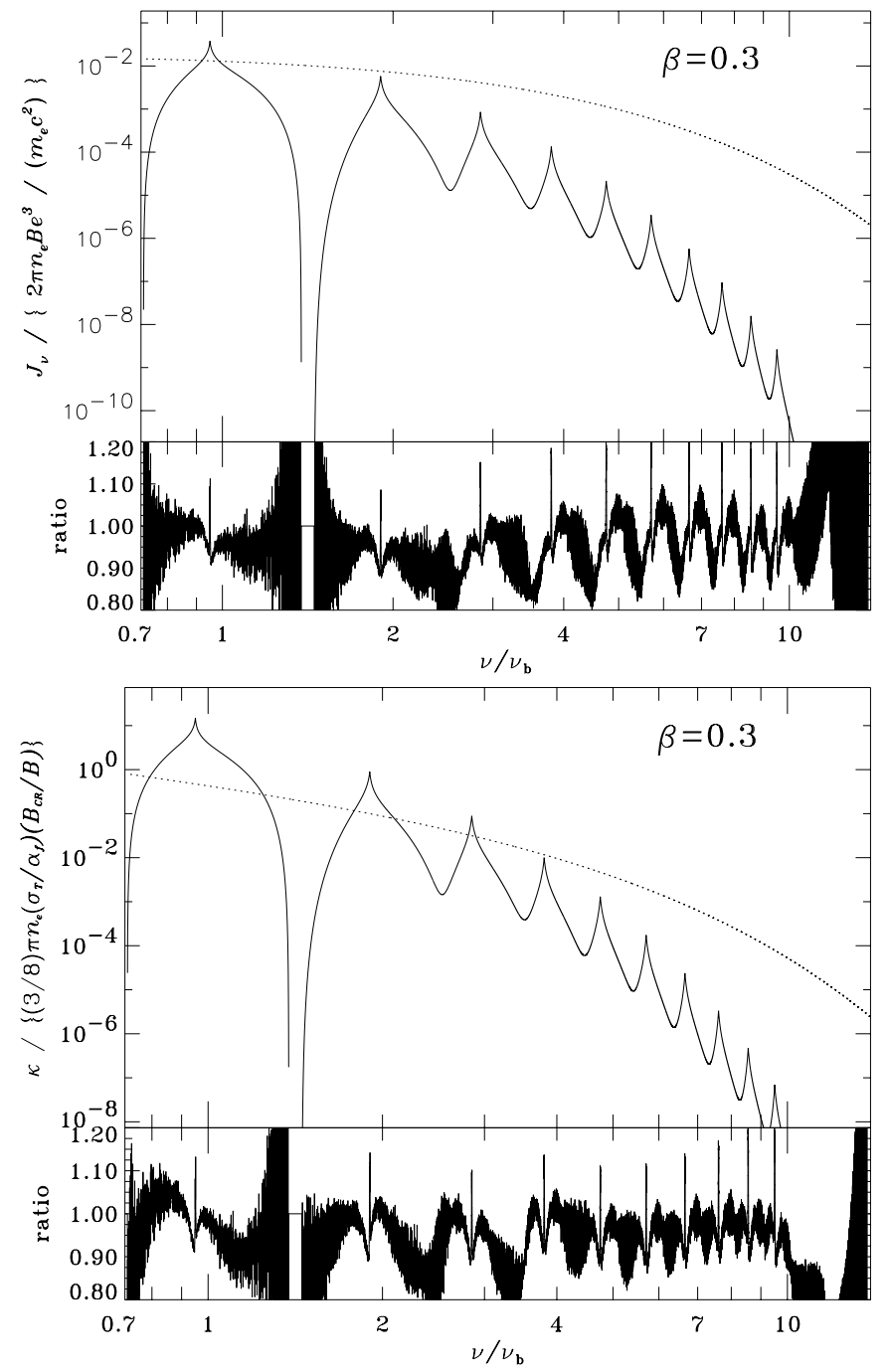

Fig. 6. Same as in Fig. 5 but for $\beta=0.3$. The formula (2.16) of GS91 in the relativistic limit for the emission coefficient is plotted in dotted line in the upper panel. The relativistic formula (2.17) of GS91 in the relativistic limit for the absorption cross-section has been used to derive the absorption coefficient plotted in dotted line in the lower panel.

For $v_{2}<v$ :

$$
\begin{aligned}
F(n, \Theta, v)= & N_{2}\left(\frac{v}{v_{2}}\right)^{-p_{6}} \frac{x_{2}-1+p_{7} \sqrt{\Theta}}{x-1+p_{7} \sqrt{\Theta}} \\
& \times \exp \left[-\frac{p_{8}}{2 \Theta}\left(\frac{1}{x}-\frac{1}{x_{2}}+x-x_{2}\right)\right] .
\end{aligned}
$$

This fitting function thus depends on 12 parameters $\left(v_{\max }, N_{\max }\right.$, $N_{1}, N_{2}$ plus 8 parameters $p_{x}$ ) that were determined from fits to our tabulated emissivities. We then constructed a table giving the best fit parameters as a function of temperature and harmonic numbers. Contrary to the case of the non-thermal emission, we have not been able to find simple fitting functions approximating conveniently the temperature dependence of these parameters (the second fitting procedure described in Sect. 5). However, the table of parameters we derived may be used to directly estimate the thermal synchrotron emission and absorption coefficients at low photon frequencies. We provide
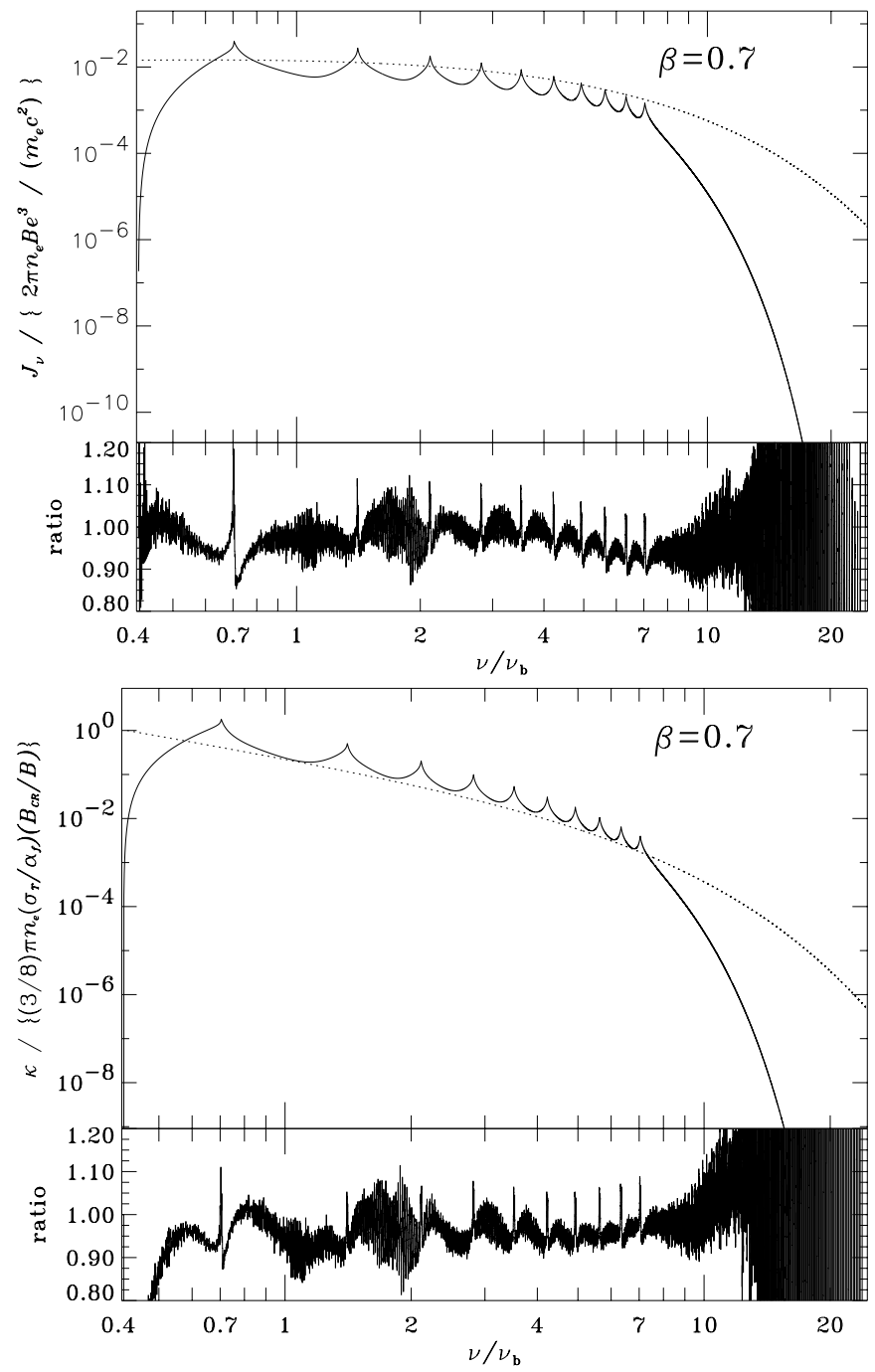

Fig. 7. Same as in Fig. 6 but for $\beta=0.7$.

electronically the parameter table together with a fortran routine computing the coefficient from that table. This methods is computationally faster and much less memory consuming than directly interpolating between the tabulated emissivities. The solutions presented here are much more accurate than the parametrised functions obtained by different authors (MNY96, WZ00 and references therein) at low temperatures and photon frequencies for which the errors around $v_{\mathrm{b}}$ are particularly severe.

\subsection{Results}

Figures 9, 10 and 11 compare the emission coefficient in $\mathrm{erg} \mathrm{s}^{-1} \mathrm{~Hz}^{-1} \mathrm{~cm}^{-3} \mathrm{st}^{-1}$ obtained using the fitting functions with the results of the numerical integration at different temperatures. The approximation appears to be good for the whole range of temperatures. For illustration we also show in these figures the results of the analytic approximation given by WZ00. As long as $\Theta<1$ this analytical estimate connects smoothly with our results around $v \sim 10 v_{\mathrm{b}}$ and may be used to compute the emission at higher frequencies. On the other 

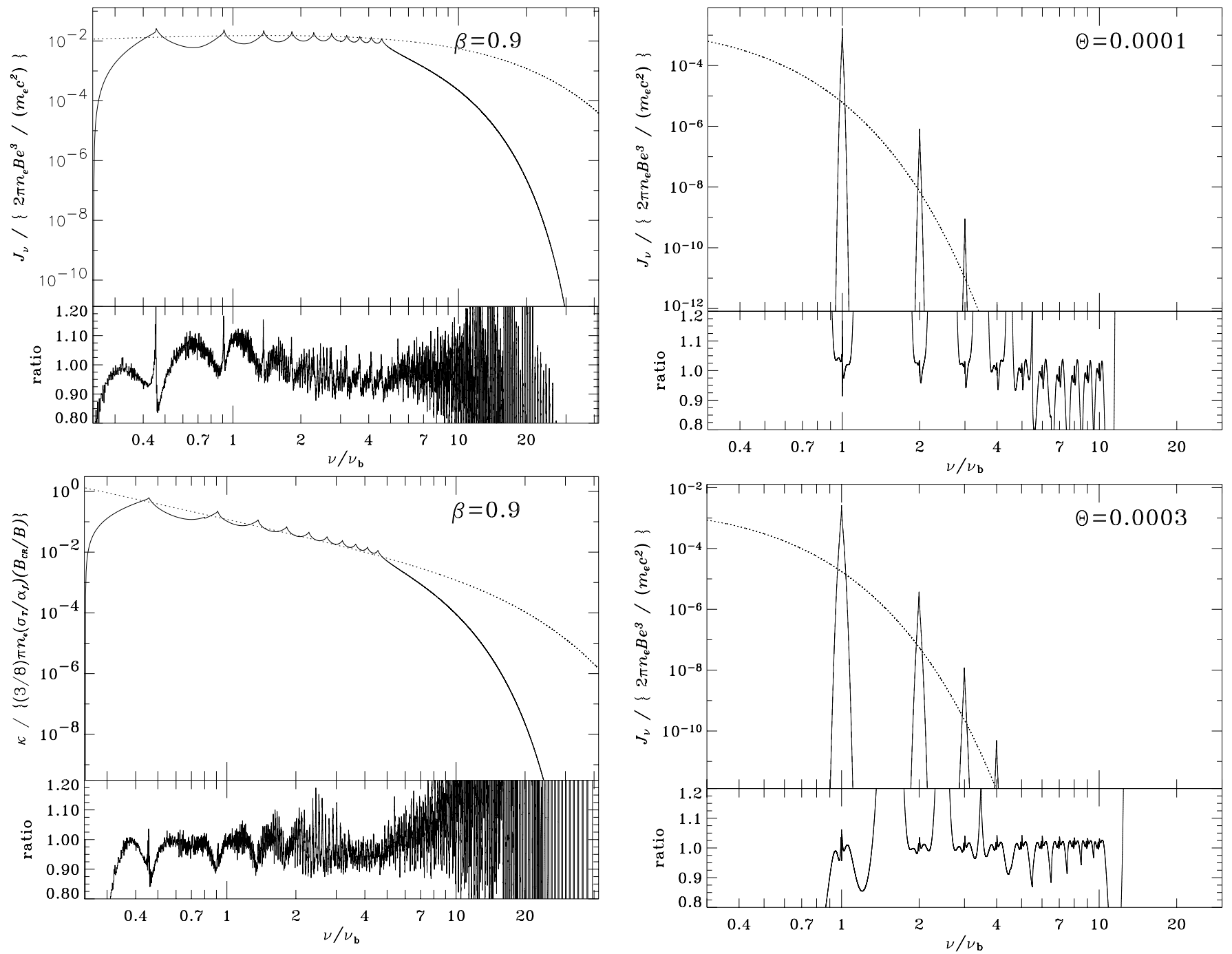

Fig. 8. Same as in Fig. 6 but for $\beta=0.9$.

hand, for $\Theta \sim 1$ and higher, the approximation of WZ00 is not so good, and our approach based on individual harmonics becomes inaccurate around $v \sim 10 v_{\mathrm{b}}$ due to the significant contribution from higher orders.

\section{Conclusion}

In this work, we presented two independent methods to compute the cyclo-synchrotron emission and absorption coefficients in a tangled magnetic field in the non- and trans relativistic regime. These calculations were done both for mono-energetic and thermal electrons. Only the first ten harmonics dominating the flux in theses regimes have been considered, but the procedure can easily be extended to a larger number of harmonics. We found synthetic fitting functions that reproduce our numerical results with a good accuracy (with a relative error at worst 20\%). They complement the previously existing approximations valid only at higher photon and electron energies. Although these fitting functions are probably too complicated to be used in analytic calculations, they may prove very useful for numerical purposes. For instance, they could

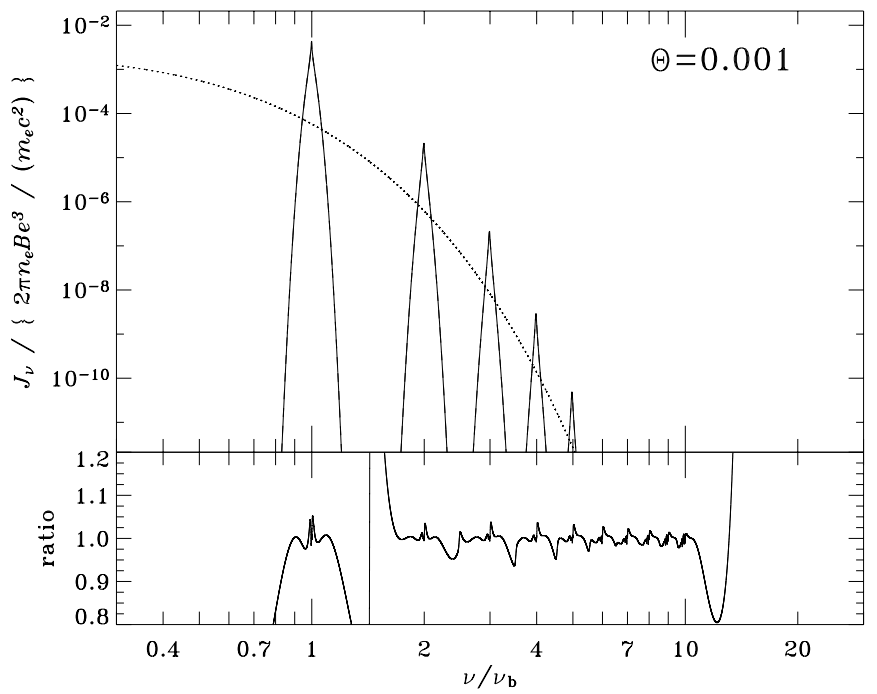

Fig. 9. From top to bottom: Thermal synchrotron emission coefficients for $k T_{\mathrm{e}}=5.36 \times 10^{-2}, 0.153$ and $0.511 \mathrm{keV}$. Upper panels: the solid curves show the approximation using the fitting functions described in Sect. 6. The results from numerical integration are indiscernable. The dotted curves show the results from the formula of WZ00. Lower panels: ratio of the fitting functions to the numerical results. 

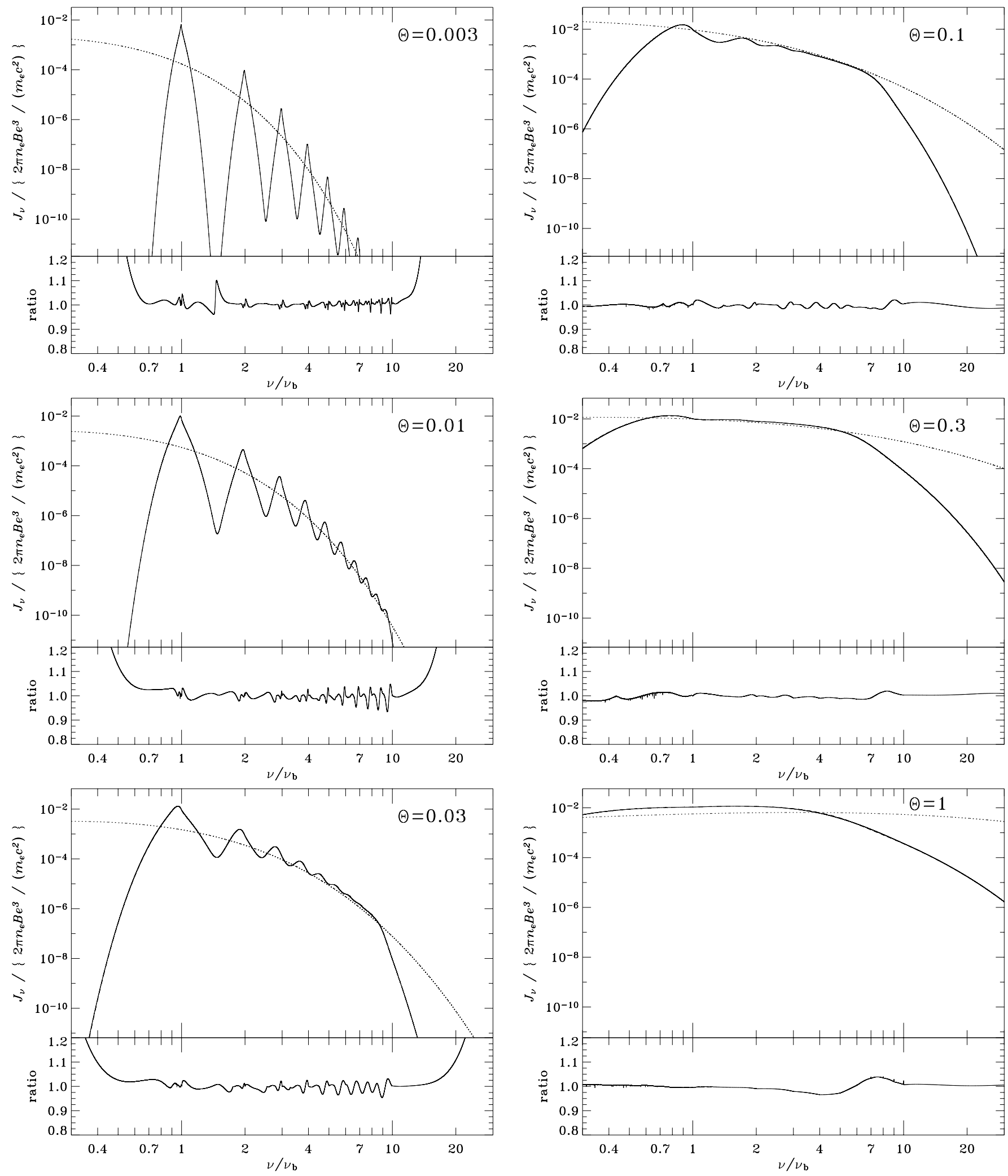

Fig. 10. Thermal synchrotron emission coefficient for $k T_{\mathrm{e}}=1.53,5.11$ and $15.3 \mathrm{keV}$ from top to bottom respectively. See caption of Fig. 9.

photon and particle dynamics (e.g. Coppi 1992; Stern et al. 1995). The fitting functions are made available electronically in the form of tables and fortran routines that can be easily implemented in such codes.
Fig. 11. Thermal synchrotron emission coefficient for $k T_{\mathrm{e}}=51.1,153$ and $511 \mathrm{keV}$ from top to bottom respectively. See caption of Fig. 9.

Acknowledgements. J.M. acknowledges financial support from the MURST (grant COFIN98-02-15-41), the European Commission (contract number ERBFMRX-CT98-0195, TMR network "Accretion onto 
black holes, compact stars and protostars"), and PPARC. The authors thank N.A. Webb for a careful reading of the manuscript.

\section{References}

Bekefi, G. 1966, Radiation processes in Plasmas (New York: Wiley) Chanmugam, G., Barrett, P. E., Wu, K., \& Courtney, M. W. 1989, ApJS, 71, 323 (C89)

Coppi, P. 1992, MNRAS, 258, 657

Di Matteo, A. Cellotti, A., \& Fabian, A. C. 1997, MNRAS, 291, 805

Ghisellini, G., \& Svensson, R. 1991, MNRAS, 252, 313 (GS91)
Ghisellini, G., Haardt, F., \& Svensson, R. 1998, MNRAS, 297, 348 (GHS98)

Ginzburg, V. L., \& Syrovatskii, S. I. 1969, ARA\&A, 7, 375

Gliozzi, M., Bodo, G., Ghisellini, G., \& Trussoni, E. 1996, MNRAS, 280, 1094

Mahadevan, R., Narayan, R., \& Yi, I. 1996, ApJ, 465, 327 (MNY96) Mason, A. 1992, MNRAS, 255, 203

Stern, B. E., Begelman, M. C., Sikora, M., \& Svensson, R. 1995, MNRAS, 272, 291

Wardziński, G., \& Zdziarski, A. A. 2000, MNRAS, 314, 183 (WZ00)

Wardziński, G., \& Zdziarski, A. A. 2001, MNRAS, 325, 963 